\title{
A COMPARATIVE ANALYSIS OF MODERN AND TRADITIONAL MEDICINE
}

\section{Prakash Rao *}

The term "traditional medicine" (TM) refers to ways of protecting and restoring health that existed before the arrival of modern medicine. As the term implies, these approaches to health belong to the traditions of each country, and have been handed down from generation to generation. Traditional medicines (TM) have met needs of the local communities for centuries. Now the terms such as alternative medicine complimentary medicine are used interchangeably to refer the traditional medicine all over the world. Despite derived from diverse cultures, Languages, Geographical locations and health beliefs of people all over the world, the common specialty and peculiarity that unites all types of alternate systems is "Holistic approach"

In 21st millennium, medical science has developed in a rapid manner particularly in the field of evidence based research. At the same time a genuine interest in various traditional practices subsists among practitioners of modern medicine along with the growing numbers of practitioners of traditional systems, acquainted with the modern technology. This kind of integration facilitates and fosters teamwork among all categories of medical professional to cater the requirements in primary health and promotes research in diverse facets for nerve-racking medical problems.

In developing countries including India, the work force represented by practitioners of traditional medicine is a potentially important resource for the delivery of health care. The World Health organization (WHO) supported every effort to formulate national policies, potential usefulness of traditional medicine as well as to evaluate the safety and efficacy of remedies to study and upgrade the knowledge of traditional medicines among modern health practitioners.

Traditional medicines, despite their limitations, are addressing health needs of millions of people worldwide. It is estimated that about $65-85 \%$ of the world population uses traditional medicines for their primary health care. It is also estimated that about 39\% of all 520 newly approved drugs in 1983-1994 were natural products and out of that 74\% were discovered as a result of bio-prospecting from plants used in traditional medicines. Traditional medicines are increasingly getting more popular mainly because (a) it is holistic system with fewer side effects (b) it is evolving as an evidencebased medicine and (c) its ethno-medical knowledge is applicable to modern drug discovery programs. It is found that there are many diseases that cannot be cured by the existing drugs and the incidence of drug resistance is increasing, consequently there is urgent need for drugs that are effective against these pathogens. Probably, traditional medicines can provide a solution in fighting them as a health care delivery mechanism as well as in exploring the medical research. As the emergence and resurgence of Traditional medicines (TM) in health care is evident worldwide. This paper attempts to make a comparative analysis to find out the current state of traditional medicine in India with respect to infrastructure development such as educational institutes, organizations, practices and therapeutic scope attitude towards alternate medicine with its limitation and implications in the field of health and medicine.

Keywords : World health organization (WHO), Traditional medicine (TM), Complimentary medicine (CAM) Holistic System,

\footnotetext{
* Prof. M. Prakash Rao, Scientist IV,

Central Council for Research in Homeopathy, New Delhi
} 